
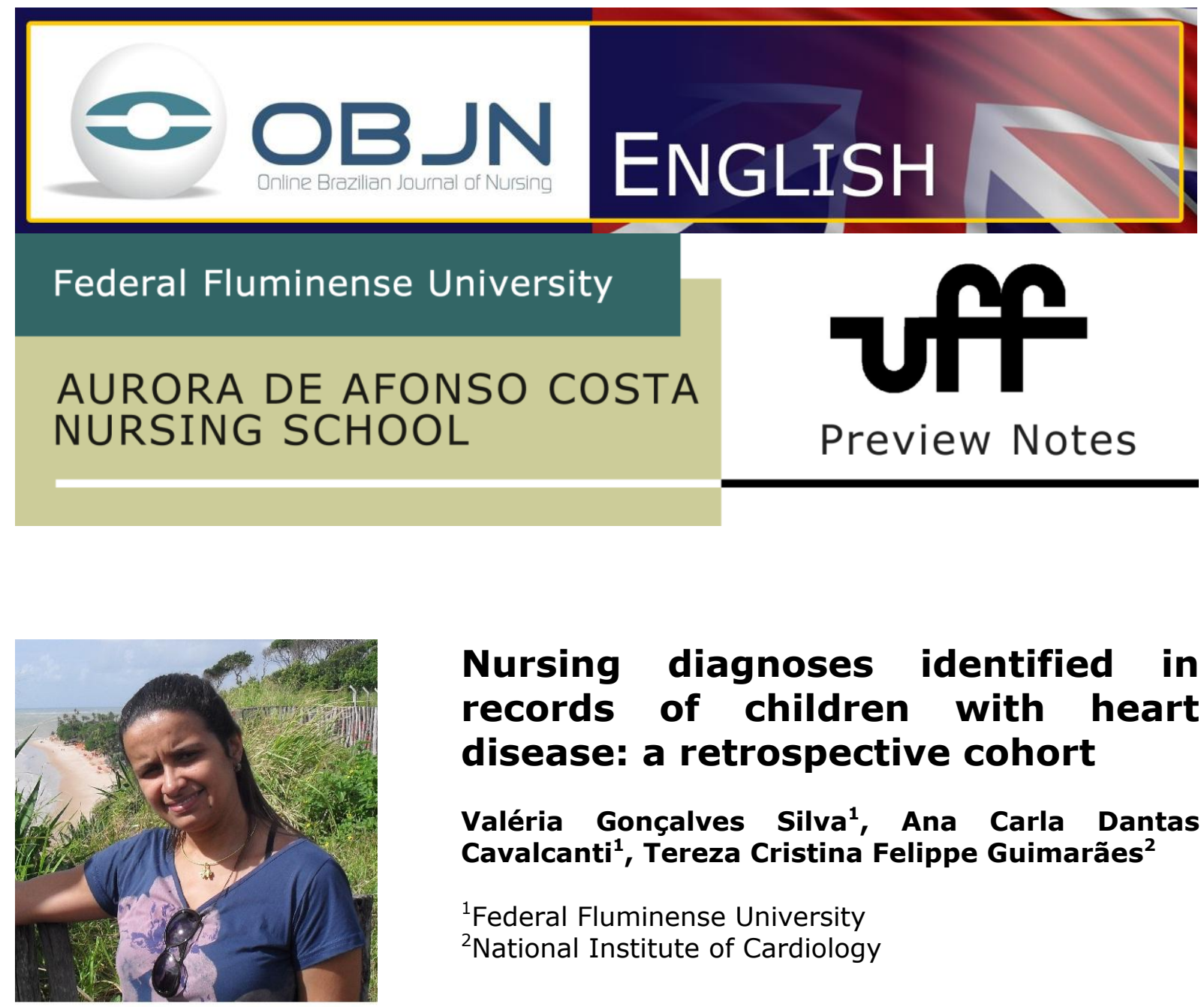

\title{
Nursing diagnoses identified in records of children with heart disease: a retrospective cohort
}

\author{
Valéria Gonçalves Silva ${ }^{1}$, Ana Carla Dantas \\ Cavalcanti $^{1}$, Tereza Cristina Felippe Guimarães ${ }^{2}$ \\ ${ }^{1}$ Federal Fluminense University \\ ${ }^{2}$ National Institute of Cardiology
}

\begin{abstract}
The dissertation for the Professional Master in Nursing degree, aims to: identify the nursing diagnoses of NANDA-I from the terms found in the nursing records of children with congenital heart disease; identify the results and nursing interventions for these diagnoses, and develop a standard nursing care instrument guided by the NANDA-NOCNIC classifications. Method: A retrospective cohort consisting of a non-probabilistic sample of convenience limited to a time frame of 6 months was studied using cross mapping as a methodological tool. It was performed through the transcription and analysis of 3 nursing records, at admission, after 24 and 48 hours, in the medical records of children with heart disease, in a federal hospital in the city of Rio de Janeiro. Results: The data obtained from medical records were submitted to descriptive analysis and agreement between the researcher and expert nurses; it was measured using the Kappa coefficient.
\end{abstract}

Keywords: Heart Defects Congenital; Nursing Diagnosis; Child. 


\section{PROBLEM SITUATION AND ITS SIGNIFICANCE}

Heart defects are the single most common congenital anomaly, and are responsible for $3 \%$ to $5 \%$ of deaths during the neonatal period. Its identification, as early as possible, is essential, given the prognostic implications and due to the incidence of fast clinical deterioration and high mortality ${ }^{(1)}$. The nursing care provided to a child with heart disease should be planned and implemented as soon as the condition is suspected for a cardiac congenital disease. For the development of the care plan, a rigorous survey of the information is essential, directed, mainly, with regard to the evaluation of the cardiac function and the identification of the child's response to heart disease complications ${ }^{(2)}$.

The nursing classifications are of great importance for the development of the profession because they are tools to compare overall knowledge. In addition, if the language used in practice is structured, standardized and classified, this will both facilitate understanding on the part of the professionals in terms of monitoring the quality of nursing care to patients, and may also help in the production of studies and scientific research ${ }^{(3)}$.

Despite difficulties in the use of standardized language when it comes to nursing hospitalized children with congenital heart disease, nurses use clinical reasoning to implement care. However, because they are not using a standard procedure for diagnoses, interventions and results, this does not provide clear answers to the situation these children are in, either in terms of implementing therapy, decision making, or the planning and evaluation of results. The standardization of language, through the nursing diagnostic classifications (NANDA-I), nursing results (NOC) and nursing interventions (NIC) are fundamental to the description of the care of patients, and is the basis for its guidance.

\section{AIM}

\section{General:}


To develop a protocol for nursing care with regard to children with congenital heart disease from their responses to the hospital routine, based on the standardized languages provided by NANDA-NOC-NIC.

\section{Specific:}

To identify nursing diagnoses from NANDA-I from the terms found in the nursing records of children with congenital heart disease;

To develop a tool for the standardization of nursing care guided by the NANDA-NOC-NIC classifications.

\section{METHOD}

A retrospective cohort study was undertaken using a cross mapping method. This involved an analysis of the nursing records of children with congenital heart disease. These children were up to two years of age, who had been hospitalized for surgical or clinical treatment for a period of less than 72 hours in the Children and Adolescents' Cardiology Service of the National Institute of Cardiology in the municipality of Rio de Janeiro. A non-probabilistic limited convenience sample that fulfilled the inclusion criteria was used for data collection between January and June 2011. Records that were not found in the medical files, those showing records which were unreadable, and those of children with congenital heart disease who have undergone corrective cardiac surgery were not used. To facilitate the analysis, mapping completion was undertaken using a registration and documentation guide. The guide consisted of five parts: a description of the child, a transcription of the records, a completion of terms extracted from the records, titles of nursing diagnoses and the evaluation of experts. The analysis was performed through a transcription and analysis of the record of admission and the twicedaily daily nursing records, following the sequence of days. The terms which describe the nursing diagnoses and which were compared with the terms of the nursing diagnoses classification from NANDA-I, were extracted for the nursing records so that any nonstandard data could be mapped into a standardized language. The mapping was done 
using an exact concordance analysis (word for word) or partial analysis (concordance with the concepts involved), and the terms were found with their defining characteristics or risk factors. Then, the real or risk diagnoses from the classification system mentioned above were identified. The whole process of cross-mapping was reviewed and refined by three expert nurses. A descriptive analysis of the diagnoses and demographic data of children with congenital heart disease (age, gender and pathology) was developed using absolute frequencies and percentages. To assess the degree of agreement between the expert nurses, the researcher used the Kappa coefficient. The knowledge of the most frequent nursing diagnoses, according to the responses of children with congenital heart disease enabled the identification of positive results and appropriate interventions. Thus, the protocol allowed the implementation of nursing care. The study was approved by the Research Ethics Committee (CEP) of the National Institute of Cardiology under No. 0355/26-10-2011.

\section{REFERENCES}

1. 1. Santana MVT. Cardiopatias Adquiridas no Recém-Nascido. São Paulo: Atheneu; 2000.

2. Silva VM, Araújo TL, Lopes MVO. Evolução dos Diagnósticos de Enfermagem de crianças com cardiopatias Congênitas. Rev Latino-Am Enfermagem. 2006; 14(4):2.

3. Nóbrega MML, Garcia TR, Nóbrega RV, Araújo TM. Bank of Terms of the nursing special language in a Neonatal Intensive Care Unit of a teaching hospital- descriptive study. Online Brazilian Journal Nursing [serial in the internet]. 2009 [cited 2011 Jul 06]; 8(2). Available http:www.objnursing.uff.br/index.php/nursing/article/view/j.16764285.2009.2321/498.

Mailing address: Rua Uruguai no 74 apto 406 - Andaraí RJ. CEP 20.510-060. E-mail: valeria.nobrega@yahoo.com.br

Valéria Gonçalves da Silva - National Institute of Cardiology, National Cancer Institute and Aurora de Afonso Costa Nursing School - Federal Fluminense University - researcher Ana Carla Dantas Cavalcanti - Aurora de Afonso Costa Nursing School - Federal Fluminense University - Guidance

Tereza Cristina Felippe Guimarães - National Institute of Cardiology - Guidance 
Approved: 03/05/2012

Received: 04/09/2012

Silva VG, Cavalcanti ACD, Guimarães TCF. Nursing diagnoses identified in records of children with heart disease: a retrospective cohort. Online braz j nurs [periodic online]. 2012 Oct [cited 2012 Oct 30]; 11 Suppl 1: 448-52. Available from: http://www.objnursing.uff.br/index.php/nursing/article/view/3901 